INPLASY

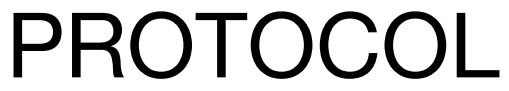

To cite: Zou et al. Postnatal prognosis of fetus with mild and moderate isolated lateral ventriculomegaly: A systematic review and meta-analysis. Inplasy protocol 2021120078. doi:

10.37766/inplasy2021.12.0078

Received: 17 December 2021

Published: 17 December 2021

Corresponding author:

Shanling Liu

sunny630_hx@163.com

Author Affiliation:

West China Second University

Hospital, Sichuan University.

Support: National

2021 YFC1005303.

Review Stage at time of this submission: Piloting of the study selection process.

Conflicts of interest:

None declared.

\section{Postnatal prognosis of fetus with mild and moderate isolated lateral ventriculomegaly: A systematic review and meta-analysis}

\author{
Zou, J1; Chen, H²; Yang, S3; Zhang, Y4; He, Y5; Xiao, X6; Liu, S7.
}

Review question / Objective: What is the postnatal prognosis of fetus with mild and moderate isolated lateral ventriculomegaly?

Condition being studied: Isolated ventriculomegaly was defined as no associated with other brain and extra-brain ultrasound abnormalities, no chromosomal abnormalities and congenital infections prenatal. Mild ventriculomegaly is defined as the width of the lateral ventricle range from 10 to $12 \mathrm{~mm}$, moderate ventriculomegaly is range from 13 to $15 \mathrm{~mm}$. The isolated mild and moderate postnatal ventriculomegaly has a prevalence of $0.7 \%$. It is reported that the moderate isolated ventriculomegaly has the poor prognosis than mild ones, but there is no meta-analysis about it. This systematic review and meta-analysis will focus on mild and moderate isolated fetal ventriculomegaly. More specifically, we would like to further stratified analysis the association between fetal isolated ventriculomegaly and postnatal outcomes. The main outcome is neurodevelopment delay/severe delay rate.

INPLASY registration number: This protocol was registered with the International Platform of Registered Systematic Review and Meta-Analysis Protocols (INPLASY) on 17 December 2021 and was last updated on 17 December 2021 (registration number INPLASY2021120078).

\section{INTRODUCTION}

Review question / Objective: What is the postnatal prognosis of fetus with mild and moderate isolated lateral ventriculomegaly?

Condition being studied: Isolated ventriculomegaly was defined as no associated with other brain and extra-brain ultrasound abnormalities, no chromosomal abnormalities and congenital infections prenatal. Mild ventriculomegaly is defined as the width of the lateral ventricle range from 10 to $12 \mathrm{~m} \mathrm{~m}$, moderate ventriculomegaly is range from 13 to $15 \mathrm{~mm}$. The isolated mild and moderate postnatal ventriculomegaly has a 
prevalence of $0.7 \%$. It is reported that the moderate isolated ventriculomegaly has the poor prognosis than mild ones, but there is no meta-analysis about it. This systematic review and meta-analysis will focus on mild and moderate isolated fetal ventriculomegaly. More specifically, we would like to further stratified analysis the association between fetal isolated ventriculomegaly and postnatal outcomes. The main outcome is neurodevelopment delay/severe delay rate.

\section{METHODS}

Search strategy: We will search the following databases: MEDLINE, EMBASE, the Cochrane Library. Combinations of the relevant medical subject heading (MeSH) terms, key words, and word variants for "Fetal", "Ventriculomegaly" Limitations: Human studies and English language literature only.

Participant or population: Fetuses with isolated fetal ventriculomegaly.

Intervention: Mild isolated ventriculomegaly, moderate isolated ventriculomegaly.

\section{Comparator: Healthy fetuses.}

Study designs to be included: Prospective cohort studies, retrospective cohort studies, cross-sectional studies and casecontrol studies.

Eligibility criteria: Inclusion - Mild ventriculomegaly was defined as an atrial measurement of $10-12 \mathrm{~mm}$; mmoderate ventriculomegaly was defined as an atrial measurement of $13-15 \mathrm{~mm}$; Isolated mild and moderate ventriculomegaly detected prenatally. Isolated (idiopathic) mild/ moderate ventriculomegaly was defined as $\mathrm{mild} / \mathrm{moderate}$ ventriculomegaly not associated with other structural abnormalities, abnormal karyotype or congenital infection. Neurodevelopmental delay was defined as abnormal neurodevelopmental score, according to the test used.
Information sources: 1.MEDLINE, EMBASE, the Cochrane Library 2.Searching other resources: Manual searches will include reviewing reference lists of identified studies and journals. Meanwhile, grey literature will be searched. And we will contact corresponding authors to identify extra studies if necessary.

Main outcome(s): Neurodevelopment outcome.

Additional outcome(s): Association with the site of the ventriculomegaly (unilateral or bilateral); Association with the intrauterine progression; Association with the region; Association with the era; Association with the development evaluation method.

Quality assessment / Risk of bias analysis: A study quality assessment will be carried out by two authors using the NewcastleOttawa Scale (NOS).

Strategy of data synthesis: We will use meta-analyses of proportions to combine data. Between-study heterogeneity will be explored using the I-squared statistic

Subgroup analysis: Considering significant heterogeneity, we plan to carry out a subgroup analysis. The following items will be considered: (1) the degree of the ventriculomegaly; (2) the site of the ventriculomegaly, unilateral or bilateral; (3) the intrauterine progression, (4)the region; (5)the era; and (6) the development evaluation method.

Sensitivity analysis: Sensitivity analysis will be conducted to eliminate the efficacy of low quality studies, provided there is significant heterogeneity after robust subgroup analysis. The meta-analysis will be repeated after low-quality studies are removed. We will compare the results of the two meta-analyses and then decide whether to exclude low-quality studies based on evidence strength, sample size, and influence on the pooled estimate.

Country(ies) involved: China. 
Keywords: Fetal isolated ventriculomegaly, neurodevelopment delay, mild, moderate.

Contributions of each author:

Author 1 - Juan Zou.

Author 2 - Huiling Chen.

Author 3 - Shuqi Yang.

Author 4 - Yuanchuan Zhang.

Author 5 - Yazhou He.

Author 6 - Xue Xiao.

Author 7 - Shanling Liu. 